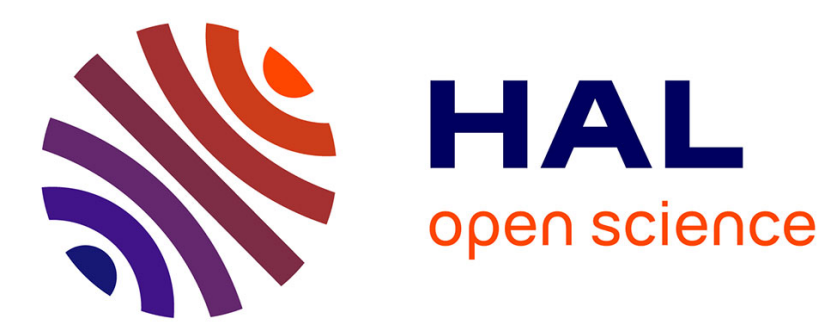

\title{
Cubature versus Fekete-Gauss nodes for spectral element methods on simplicial meshes
}

\author{
Richard Pasquetti, Francesca Rapetti
}

\section{To cite this version:}

Richard Pasquetti, Francesca Rapetti. Cubature versus Fekete-Gauss nodes for spectral element methods on simplicial meshes. Journal of Computational Physics, 2017. hal-01589136

\section{HAL Id: hal-01589136 \\ https://hal.univ-cotedazur.fr/hal-01589136}

Submitted on 18 Sep 2017

HAL is a multi-disciplinary open access archive for the deposit and dissemination of scientific research documents, whether they are published or not. The documents may come from teaching and research institutions in France or abroad, or from public or private research centers.
L'archive ouverte pluridisciplinaire HAL, est destinée au dépôt et à la diffusion de documents scientifiques de niveau recherche, publiés ou non, émanant des établissements d'enseignement et de recherche français ou étrangers, des laboratoires publics ou privés. 


\title{
NOTE \\ Cubature versus Fekete-Gauss nodes for spectral element methods on simplicial meshes
}

\author{
Richard Pasquetti ${ }^{1}$, Francesca Rapetti \\ Université Côte d'Azur, CNRS, Inria, Lab. J. A. Dieudonné, F-06108 Nice, France.
}

Keywords:

spectral element method; triangular elements; diagonal mass matrix; cubature points; Fekete points; Fekete-Gauss approximation.

In a recent JCP paper [9], a higher order triangular spectral element method (TSEM) is proposed to address seismic wave field modeling. The main interest of this TSEM is that the mass matrix is diagonal, so that an explicit time marching becomes very cheap. This property results from the fact that, similarly to the usual SEM (say QSEM), the basis functions are Lagrange polynomials based on a set of points that shows both nice interpolation and quadrature properties. In the quadrangle, i.e. for the QSEM, the set of points is simply obtained by tensorial product of Gauss-Lobatto-Legendre (GLL) points. In the triangle, finding such an appropriate set of points is however not trivial. Thus, the work of [9] follows anterior works that started in 2000's $[2,6,11]$ and now provides cubature nodes and weights up to $N=9$, where $N$ is the total degree of the polynomial approximation. Here we wish to evaluate the accuracy of this cubature nodes TSEM with respect to the Fekete-Gauss one, see e.g. [12], that makes use of two sets of points, namely the Fekete points and the Gauss points of the triangle for interpolation and quadrature, respectively. Because the Fekete-Gauss TSEM is in the spirit of any nodal $h p$-finite element methods, one may expect that the conclusions of this Note will remain relevant if using other sets of carefully defined interpolation points.

First, recall that if two different sets of points are used for interpolation and quadrature, then the space $\mathbb{P}_{N}(\hat{T})$ of polynomials of maximal (total) degree $N$, defined on the reference triangle $\hat{T}=\{(r, s)$ : $r \in(-1,1), s \in(-1,-r)\}$, is usually used as approximation space. The cardinality of this space equals $n=(N+1)(N+2) / 2$, that can be associated to $n$ interpolation points if using Lagrange polynomials as basis functions. If 3 of these nodes coincide with the vertices of the element, then $3 N$ of these $n$ points should belong to the edges of $\hat{T}$ and the remaining $(N-1)(N-2) / 2$ are the inner nodes. Usually, the edge nodes proposed in the literature coincide with the GLL points, see e.g. $[1,5,15]$. Since one does not know an explicit formulation of the Lagrange basis functions, say $\varphi_{i}(r, s), 1 \leq i \leq n$, to compute their values or those of their derivatives at a given point one generally makes use the orthogonal Kornwinder-Dubiner (KD) basis [4], for which explicit formula exist. Gauss points for the triangle and the corresponding quadrature formula may be found in the literature, up to degree $M \approx 20$ if a symmetric distribution of the points is desired [3]. In practice, one may choose $M=2 N$, so that both the stiffness and mass matrix are exactly computed, since their entries are polynomials of degree $2 N$ and $2 N-2$, respectively. For details on the implementation of the Fekete-Gauss approach, see e.g. [12].

If using a single set of points, as just mentioned, $3 N$ of the interpolations points must belong to the triangle boundary with 3 of them at the vertices. As demonstrated in $[7,16]$, such a strong constraint forbids the possibility of finding a set of cubature points providing a sufficiently accurate cubature formula, if looking for basis functions that span the space $\mathbb{P}_{N}$. To overcome this difficulty, as first developed in [2], the idea is then to enrich the space $\mathbb{P}_{N}$ by polynomial bubble functions of degree $N^{\prime}>N$. This indeed allows to

\footnotetext{
${ }^{1}$ Corresponding author. E-mail address: richard.pasquetti@unice.fr
} 
include new cubature points inside the triangle while keeping the element boundary nodes number equal to $3 N$. In the reference triangle $\hat{T}$, this may be achieved by introducing the polynomial space $\mathbb{P}_{N} \cup b \times \mathbb{P}_{N^{\prime}-3}$, where $b$ is the (unique) bubble function of $\mathbb{P}_{3}(\hat{T})$, namely $b(r, s)=(r+1)(s+1)(r+s)$. The cardinality of this space then equals $n^{\prime}=3 N+\left(N^{\prime}-1\right)\left(N^{\prime}-2\right) / 2$. Now, to compute the Lagrange polynomials at a given points one should use an extended KD basis, composed of the usual KD basis of $\mathbb{P}_{N}$ completed by those KD polynomials of $\mathbb{P}_{N^{\prime}-3}$ which once multiplied by the bubble function $b$ are of degree strictly greater than $N$. Of course, $N^{\prime}$ should be chosen as small as possible to avoid a useless increase of the inner nodes number: In [2] and posterior works, $N^{\prime}$ is chosen such that it exists a cubature rule exact for polynomials of degree $N+N^{\prime}-2$. The determination of $N^{\prime}$, together with the cubature points and weights gives rise to a difficult optimization problem, see [9] for details. It turns out that $N^{\prime}-N$ increases monotonically with $N: N^{\prime}=N+1$, for $1<N<5, N^{\prime}=N+2$ for $N=5$ and $N^{\prime}=N+3$ for $5<N<10$. It should be noticed that with the cubature nodes based TSEM, neither the mass matrix nor the stiffness matrix are exactly computed, since their entries are of degree $2 N^{\prime}$ and $2 N^{\prime}-2$, respectively. This is why a comparative study of the accuracy of these two different TSEMs is of interest.

Such a comparison has been carried out for elliptic problems, using the Fekete-Gauss TSEM software that we have developed for a few years. A variant of this code has been easily implemented for the cubature points based TSEM: Indeed, using a single set of points provides a simplification and extending the KD basis only constitutes a minor task. Especially, the code makes use of the condensation technique, i.e. one first computes the unknowns associated to the edges of the elements and then reconstructs the numerical solution inside locally. Thus, the algebraic systems that result from the two TSEMs approximations are exactly of same size, and so the computational times compare very well. They are simply solved by using a standard conjugate gradient method, with Jacobi preconditionner in a matrix free implementation [8].
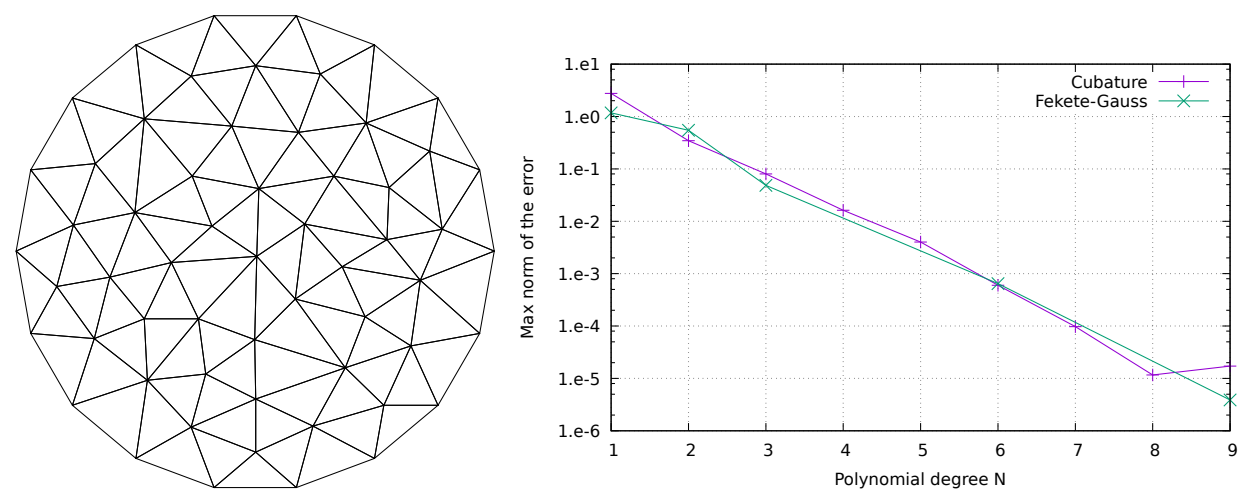

Figure 1: Mesh of the computational domain (left) and max norm of the error (right) for the Poisson equation

The comparison has first been carried out for the Poisson equation, using the exact solution $u_{e x}=$ $\cos (10 x) \cos (10 y)$ and the source term $f=-\Delta u_{e x}$, with a Dirichlet boundary condition. Computations have been made in a quasi-circular computational domain with the spectral element mesh shown in Fig. 1 (left). The max norm of the error, computed at the interpolation (cubature or Fekete) points, with respect to the polynomial degree is provided in Fig. 1 (right). In this semi-log scale plot, the expected exponential convergences are clearly observed. Moreover, the fact that for the cubature points based TSEM the stiffness matrix is only approximately computed has a negligible influence on the error.

Comparisons have also been carried out for the elliptic equation $-\Delta u+\sigma u=f$, with $\sigma=1$ and $\sigma=1000$. The high value of $\sigma$ is of interest when thinking to unsteady diffusion problems, for which at each time step one has to solve the equation with $\sigma$ scaling like the inverse of the time step. Here again, see Fig. 2, one observes that the cubature TSEM compares very well with the Fekete-Gauss approach. Some remarks may be expressed:

- For the smaller values of the polynomial degree $N$, the error is very large and the curves are a little bit erratic. Indeed, for small $N$ the discretization is too rough to follow the oscillations of the exact solution. 

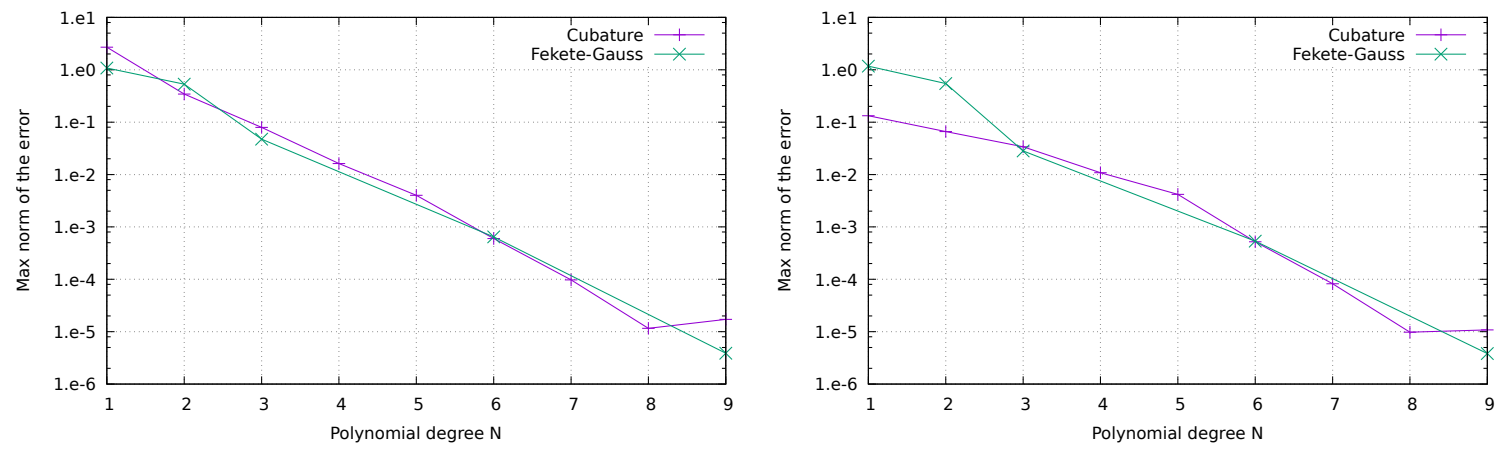

Figure 2: Max norm of the error for $\sigma=1$ (left) and $\sigma=1000$ (right)

- The two methods do not provide same errors for these small values of $N$. Indeed, the integrals are not computed in a same way and for $N=2$ the cubature based TSEM makes use of an inner point.

- For $N=9$, the cubature TSEM does not provide results better than those obtained for $N=8$. To check that, we have made computations with the polynomial solution $u_{e x}=\left(x^{2}+y^{2}\right) / 4$, which of course is of degree 2. For $2 \leq N \leq 8$ the error is close to the error associated to the iterative solve, but this is not the case for $N=9$, see Table 1 . An improvement in the determination of the cubature points and weights is here clearly needed, even if in practice a so high value of $N$ is rarely used.

\begin{tabular}{c|c|c|c|c|c}
$N$ & 2 & 6 & 7 & 8 & 9 \\
\hline Error & $1.277810^{-12}$ & $3.152310^{-12}$ & $2.845110^{-12}$ & $3.464110^{-12}$ & $1.101210^{-7}$
\end{tabular}

Table 1: Max norm of the error for the Laplace equation with exact solution of degree 2 and various $N$.

Till now a Dirichlet condition was used to compare the two different TSEMs. Addressing homogeneous Neumann conditions, as in [11], is trivial, since the boundary integral that appears in the variational formulation cancels. This is however no longer true if the Neumann boundary value is not homogeneous or more generally if a mixed (say Robin) condition should be implemented. In case of the Fekete-Gauss TSEM, the boundary integrals can be easily computed since the edge Fekete nodes coincide with the GLL points. On the contrary, the edge cubature points are not of Gauss type. In [9], where the wave equation is considered, one only makes use of Dirichlet conditions via eventually a PML (perfectly matched layer) to address the absorbing wall case. This is why it is of interest to consider again the previous elliptic problem but with now the Robin condition $\partial_{n} u+\alpha u=g$ on the boundary. Two different approaches are investigated hereafter for the cubature points TSEM.

The simplest (say naive) method consists in defining a quadrature rule based on the edge nodes. Since such a quadrature rule will be only exact for polynomials of degree $N$, one may however expect a loss of accuracy due to a not enough accurate computation of the boundary integrals of the products $\alpha \varphi_{i} \varphi_{j}$, where $\varphi_{i}$ and $\varphi_{j}$ are two of the Lagrange polynomials based on the cubature points, and $g_{N} \varphi_{i}$, with $g_{N}$ for the piecewise polynomial approximation of degree $N$ of $g$. For a more accurate method, the boundary integrals must be computed by using on each element edge of the boundary a Gauss quadrature rule, e.g. the one based on the GLL points. Since the restrictions of the Lagrange polynomials $\varphi_{i}$ at the element edges of the boundary are polynomials of degree $N$, one can span this polynomial space with the Legendre polynomials, say $L_{i}(r)$ with $r \in[-1,1]$, for the reference edge, and $0 \leq i \leq N$. Then, one can set up the Vandermonde matrices of size $(N+1) \times(N+1)$ based on the cubature and GLL points, say $V_{C u b}$ and $V_{G L L}$. One has e.g. $\left(V_{C u b}\right)_{i j}=L_{j}\left(r_{i}\right)$, with $r_{i} \in[-1,1]$ for the edge cubature points. Then, the matrix $V_{G L L} V_{C u b}^{-1}$ allows to compute at the GLL points quantities known at the cubature points. Especially, each column of this matrix provides the values of the edge Lagrange polynomials at the GLL points. Note that for straight triangular elements, the edge Jacobian determinant is constant on each edge and proportional to its length. 
On the contrary, when considering curved triangular elements, see e.g. [13], some care is needed for a relevant computation of the edge Jacobian determinants at the GLL points from those at the cubature points (the edge Jacobian determinant being then a polynomial of degree greater than $N$ ). Finally, note that for the naive approach the quadrature weights are simply proportional to the entries of the first line of the matrix $V_{C u b}^{-1}$.
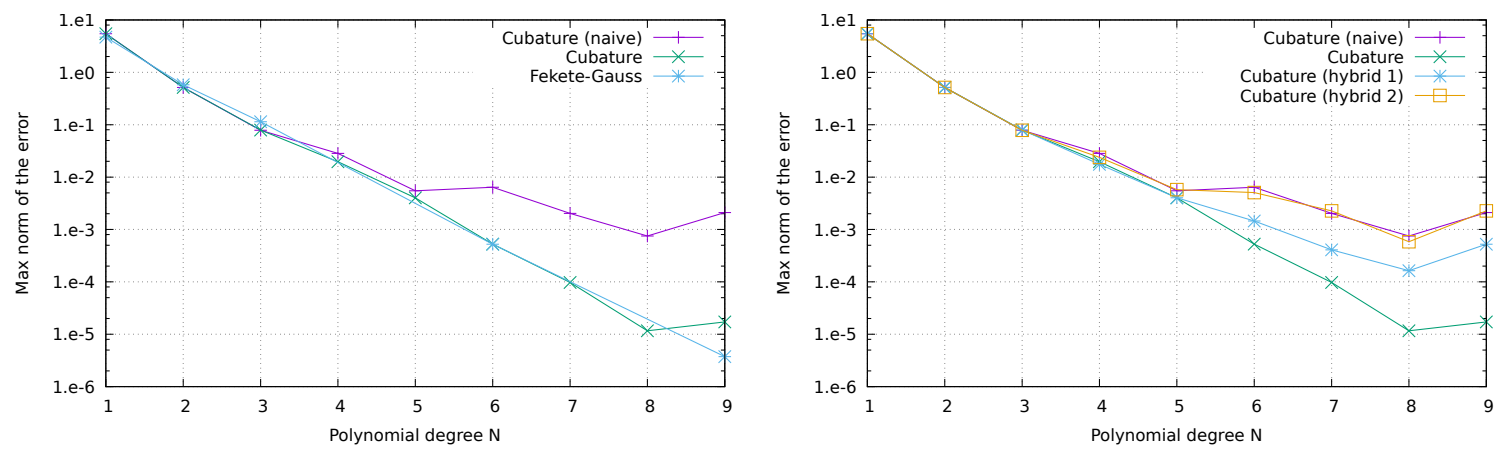

Figure 3: Max norm of the error for the Robin problem (left) and when using hybrid approaches (right).

Computations have been carried out for the equation $-\Delta u+u=f$ and the Robin condition $\partial_{n} u+u=g$, with $f$ and $g$ defined from $u_{e x}=\cos (10 x) \cos (10 y)$. In Fig. 3 (left) the results obtained with the FeketeGauss TSEM are compared to those obtained with the cubature points based one, using either the naive or the more accurate method. Clearly, when using the naive approach the spectral convergence gets lost, whereas with the accurate one the convergence curve compares quite well with the Fekete-Gauss one (for $N \leq 8)$. Does the naive approach induce a similar loss of spectral accuracy for the pure Neumann problem, i.e. with $\partial_{n} u=g$ on the boundary? The answer of course depends on the boundary data: Thus, if $g$ is piecewise constant then the boundary integrals can be exactly computed. However, for the highly oscillating $g$ associated to the present exact solution very similar results are obtained for the Robin and Neumann problems. Revisiting the Robin problem with hybrid approaches that consist of using the GLL quadrature rule only for integrating either the $g_{N} \varphi_{i}$ (hybrid 1) or the $\alpha \varphi_{i} \varphi_{j}$ (hybrid 2) kernels, one can indeed observe that here the loss of accuracy is mainly associated with the boundary data, see Fig. 3 (right).

The cubature points based TSEM was essentially developed for the wave equation. However, as soon as an explicit time stepping is involved, this TSEM is useful. In another context, the fact that the SEM mass matrix is diagonal allows to define high order differential operators and thus to address efficiently dispersive equations [10]. As a conclusion, a cubature points based TSEM may find interest in various different situations, and progresses for the tetrahedron would be welcome.

Acknowledgments: The $\mathbb{P}_{1}$ FEM mesh has been generated with the free software "Triangle". We are grateful to Dr Youshan Liu, Chinese Academy of Sciences, Beijing, for transmitting us the cubature points and weights used in [9].

\section{References}

[1] M.G. Blyth, C. Pozrikidis, A Lobatto interpolation grid over the triangle, IMA J. Appl. Math., 71 (1) (2006) $153-169$.

[2] G. Cohen, P. Joly, J.E. Roberts, N. Tordjman, Higher order triangular finite elements with mass lumping for the wave equation, SIAM J. Numer. Anal. 38 (2001) 2047-2078.

[3] R. Cools, An encyclopaedia of cubature formulas, Journal of complexity, 19 (3) (2003) 445-453.

[4] M. Dubiner, Spectral methods on triangles and other domains. J. Sci. Comput., 6 (1991) 345-390.

[5] G. Gassner, F. Lörcher, C.D. Munz, J. Hestaven, Polymorphic nodal elements for discontinuous spectralhp element methods, J. Comput. Phys., 228 (5) (2009) 1573-1590. 
[6] F.X. Giraldo, M.A. Taylor, A diagonal mass matrix triangular spectral element method based on cubature points, J. of Engineering Mathematics 56 (2006) 307-322.

[7] B.T. Helenbrook, On the existence of explicit hp-finite element methods using Gauss-Lobatto integration on the triangle, SIAM J. Numer. Anal., 47 (2009) 1304-1318.

[8] L. Lazar, R. Pasquetti, F. Rapetti, Fekete-Gauss spectral elements for incompressible Navier-Stokes flows: The twodimensional case, Comm. in Comput. Phys. 13 (2013) 1309-1329.

[9] Y. Liu, J. Teng, T. Xu, J. Badal, Higher-order triangular spectral element method with optimized cubature points for seismic wavefield modeling, J. of Comput. Phys. 336 (2017) 458-480.

[10] S. Minjeaud, R. Pasquetti, High order $C^{0}$ Galerkin schemes for high order PDEs, conservation of quadratic invariants and application to the Korteweg-De Vries model, J. of Sci. Comput., (2017) doi:10.1007/s10915-017-0455-2.

[11] W.A. Mulder, New triangular mass-lumped finite elements of degree six for wave propagation, Progress in Electromagnetic Research 141 (2013) 671-692.

[12] R. Pasquetti, F. Rapetti, Spectral element methods on unstructured meshes: comparisons and recent advances, J. Sci. Comp. 27 (1-3) (2006) 377-387.

[13] R. Pasquetti, Comparison of some isoparametric mappings for curved triangular spectral elements, J. of Comput. Phys. 316 (2016) 573-577.

[14] M.A. Taylor, B. Wingate, R.E. Vincent, An algorithm for computing Fekete points in the triangle. SIAM J. Numer. Anal., 38 (2000) 1707-1720.

[15] T. Warburton, An explicit construction for interpolation nodes on the simplex. J. Eng. Math. 56 (3) (2006) $247-262$.

[16] Y. Xu, On Gauss-Lobatto integration on the triangle, SIAM J. Numer. Anal., 49 (2011) 541-548. 